\title{
Validation and Clinical Application of a New Liquid Chromatography Coupled to Mass Spectrometry (HPLC-MS) Method for Dalbavancin Quantification in Human Plasma
}

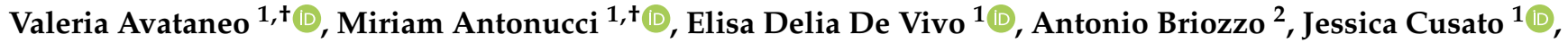 \\ Francesca Bermond ${ }^{3}$, Corrado Vitale ${ }^{3}$, Francesco Vitale ${ }^{2}$, Alessandra Manca ${ }^{1}{ }^{\mathbb{D}}$, Alice Palermiti ${ }^{1} \mathbb{D}$, \\ Giovanni Di Perri ${ }^{4}$, Francesco Giuseppe De Rosa ${ }^{4}\left(\mathbb{D}\right.$, Amedeo De Nicolò ${ }^{1, *, \ddagger(1)}$ and Antonio $D^{\prime}$ Avolio ${ }^{1, \ddagger(1)}$ \\ 1 Laboratory of Clinical Pharmacology and Pharmacogenetics, Department of Medical Sciences, \\ University of Turin, 10149 Turin, Italy; valeria.avataneo@unito.it (V.A.); \\ miriam.antonucci20@gmail.com (M.A.); elisa.devivo59@gmail.com (E.D.D.V.); jessica.cusato@unito.it (J.C.); \\ alessandra.manca@unito.it (A.M.); alice.palermiti@unito.it (A.P.); antonio.davolio@unito.it (A.D.) \\ 2 Department of Internal Medicine, A.O. Umberto I Ordine Mauriziano, 10128 Turin, Italy; \\ abriozzo@tiscali.it (A.B.); fvitale@mauriziano.it (F.V.) \\ 3 Nephrology and Dialysis, A.O. Ordine Mauriziano di Torino, 10128 Turin, Italy; \\ fbermond@mauriziano.it (F.B.); covitale@mauriziano.it (C.V.) \\ 4 Unit of Infectious Diseases, Department of Medical Sciences, University of Turin, 10149 Turin, Italy; \\ giovanni.diperri@unito.it (G.D.P.); francescogiuseppe.derosa@unito.it (F.G.D.R.) \\ check for \\ updates \\ Citation: Avataneo, V.; Antonucci, \\ * Correspondence: amedeo.denicolo@unito.it; Tel.: +39-011-4393867 \\ + Both authors contributed equally to this work. \\ $\ddagger$ Both authors contributed equally to this work.
}

M.; De Vivo, E.D.; Briozzo, A.; Cusato, J.; Bermond, F.; Vitale, C.; Vitale, F.; Manca, A.; Palermiti, A.; et al. Validation and Clinical Application of a New Liquid Chromatography Coupled to Mass Spectrometry (HPLC-MS) Method for Dalbavancin Quantification in Human Plasma. Separations 2021, 8, 189. https:// doi.org/10.3390/separations 8100189

Academic Editors: Ivan Mikšík and Caroline West

Received: 8 September 2021

Accepted: 12 October 2021

Published: 15 October 2021

Publisher's Note: MDPI stays neutral with regard to jurisdictional claims in published maps and institutional affiliations.

Copyright: (c) 2021 by the authors. Licensee MDPI, Basel, Switzerland. This article is an open access article distributed under the terms and conditions of the Creative Commons Attribution (CC BY) license (https:/ / creativecommons.org/licenses/by/ $4.0 /)$.
Abstract: Dalbavancin (DBV) is an intravenous long-acting second-generation glycolipopeptide antibiotic with high efficacy and excellent tolerability, approved for use in the treatment of Grampositive skin and skin structure infections (ABSSSI). Nevertheless, little is known about its pharmacokinetic/pharmacodynamic (PK/PD) properties in real life, which is also due to technical challenges in its quantification in human plasma, preventing an effective application of therapeutic drug monitoring (TDM). In fact, DBV has a high affinity to plasma proteins, possibly resulting in poor recovery after extraction procedure. The aim of this study was to validate a simple, cheap and reliable HPLCMS method for use in TDM, in accordance with FDA and EMA guidelines. The optimized protein precipitation protocol required $50 \mu \mathrm{L}$ of plasma, while chromatographic analysis could be performed in $12 \mathrm{~min} /$ sample. This method fulfilled the guidelines requirements and then, it was applied for routine DBV TDM in patients receiving off-label high doses (two $1500+1500 \mathrm{mg}$ weekly infusions instead of $1000+500 \mathrm{mg}$ ), with normal renal function or undergoing hemodialysis: continuous hemodiafiltration caused a relevant reduction in DBV exposure, while intermittent dialysis showed comparable DBV concentrations with those of patients with normal renal function. This confirmed the eligibility of the presented method for use in TDM and its usefulness in clinical practice.

Keywords: dalbavancin; therapeutic drug monitoring; long-acting therapy; glycopeptides; MRSA; skin infections; osteomyelitis; dialysis

\section{Introduction}

In recent years, a new lipoglycopeptide antibiotic named DBV has been approved for the treatment of ABSSSI [1]. DBV is a semi-synthetic antibiotic with a structure very similar to that of teicoplanin: the most important improvement is the addition of an extended lipophilic side chain that allows DBV dimerization and anchoring to the bacterial cell membrane, thus enhancing its potency, prolonging its half-life and allowing for extended dosing intervals [2-5].

As with other glycopeptides, DBV acts by inhibiting the formation of peptidoglycan cross-linking in Gram-positive bacteria through interaction with terminal D-alanyl-D- 
alanine residues of peptidoglycan precursors, causing a natural resistance mechanism in Gram-negative ones. This antibiotic was shown to be particularly effective even against methicillin-resistant Staphylococcus aureus (MRSA), and its breakpoint MIC (Minimum Inhibitory Concentration) for both staphylococci of various species and streptococci is reported to be $0.125 \mathrm{mg} / \mathrm{L}[6,7]$. Recent studies reported challenges related to the management of vancomycin-based treatments, including its inconvenient dosing (intravenous administration every 6 or $12 \mathrm{~h}$ ), the occurrence of side effects (in particular nephrotoxity) and the increasing emergence of MRSA strains with reduced susceptibility to this drug $[2,4,5]$; conversely, up-to-date DBV has never been related to nephrotoxity, it has proved more effective in particular circumstances (e.g., osteomyelitis [8,9]), it showed an extremely long half-life, thus allowing a weekly intravenous dosage, and demonstrated a very high AUC (Area Under the Curve)/MIC ratio, reducing the onset of resistant isolates and, sometimes, overcoming eventual resistance to vancomycin $[2,5,10,11]$.

Several studies accurately described DBV pharmacokinetics both in healthy volunteers and in patients with renal/hepatic impairment [12-15]. Assuming a common regimen consisting of a first administration of $1000 \mathrm{mg}$ on day 1 followed by a second dose of $500 \mathrm{mg}$ on day 8 [13], the expected mean $\mathrm{C}_{\max }$ after the first infusion is nearly $250 \mathrm{mg} / \mathrm{L}$ for patients with normal renal function, and it was shown to increase in the case of renal dysfunctions [14]; the same trend has been observed for $\mathrm{AUC}_{0-7 \text { days }}$ [14]. All these data reflect a $C L$ reduction in the presence of renal impairment, even more pronounced after day 7; in particular, in the case of severe renal damage, the CL is reduced by half, causing the area under curve $\mathrm{AUC}_{0-\infty}$ to double [14]. Conversely, patients who undergo regular intermittent hemodialysis (IHD) seem to not require dose adjustment $[5,14]$. Conversely, data about the impact of continuous renal replacement therapy, and in particular, veno-venous hemodiafiltration (CVVHDF) on DBV exposure are still few and controversial [16-18].

Considering the peculiar features and the evidence of an AUC/MIC-dependent effect for DBV against both staphylococci and streptococci $[11,19,20]$, TDM could be beneficial in order to optimize its clinical effectiveness.

Nevertheless, several difficulties related to the quantification of DBV in plasma samples have been previously reported: in fact, it is well known that DBV shows extensive plasma protein binding (approximately 93\%) that may cause drug co-precipitation during the extraction procedure; moreover, in the same work, a non-specific binding (NSB) to plastics has been reported [21]. Moreover, the currently published methods for DBV quantification are based on the use of highly expensive platforms and reagents, such as Ultra-High-Performance Liquid Chromatography (UHPLC) and tandem mass spectrometry (MS/MS), using isotope-labeled internal standards or analogues that are not commercially available $[12,15,21-23]$. On the other hand, some of these methods were only validated on rat plasma $[22,23]$ and/or their described validation process lacked recovery or matrix effect data $[12,15,22]$, which can be extremely critical for the ruggedness of a method, particularly considering a molecule such as DBV.

In this context, considering the challenges related to DBV determination in plasma and the wide need for a cheap yet reliable quantification method, a novel High-Performance Liquid Chromatography method coupled to single mass spectrometry (HPLC-MS) has been developed and validated in accordance with FDA and EMA guidelines [24,25] and finally applied for use in TDM on incurred samples from patients receiving an off-label posology (two intravenous infusions of $1500 \mathrm{mg}$, once weekly for two weeks, instead of the usual $1000+500 \mathrm{mg}$ schedule [26]) to treat osteoarticular infections, with normal or altered renal function.

\section{Materials and Methods}

\subsection{Chemicals}

HPLC-grade acetonitrile (ACN) and methanol $(\mathrm{MeOH})$ were purchased from VWR (Milan, Italy). HPLC-grade $\mathrm{H}_{2} \mathrm{O}$ was produced using a Milli-DI system coupled with a Synergy 185 system by Millipore (Milan, Italy). Blank plasma from healthy donors was 
acquired from the Blood Bank of ASL Città della Salute di Torino (Turin, Italy). Formic and phosphoric acids, 6,7-dimethyl-2,3-di(2-pyridyl) quinoxaline (QX) (purity 99.9\%), the Internal Standard (IS), L-arginine (purity 99\%), and Triton-X-100 were purchased from SigmaAldrich Corporation (Milan, Italy). DBV (purity 99\%) was kindly donated by CoQua $\mathrm{Lab}$ (Turin, Italy). DBV pure powder was stored at $-20{ }^{\circ} \mathrm{C}, \mathrm{QX}$ was stored at $+4{ }^{\circ} \mathrm{C}$, and $\mathrm{L}$-arginine at room temperature, in the dark in order to prevent any possible degradation.

\subsection{Preliminary Experiments}

In this work, we aimed to develop a sample preparation method with an optimized protein precipitation solution: this was performed by testing different combinations and proportions of $\mathrm{ACN}$ and $\mathrm{MeOH}$. Moreover, in order to evaluate $\mathrm{pH}$-dependent DBV solubility and protein binding, different percentages of phosphoric acid were added to the IS working solution and tested. Finally, in order to prevent DBV NSB to vial walls, both Triton-X-100 at $1 \%$ and different concentrations of L-arginine were evaluated. Optimization of the target single-ion recording (SIR) traces for DBV and IS was performed through direct continuous infusion into the MS source.

\subsection{Stock Solutions, Internal Standard, Standards, and Quality Controls}

DBV stock solution was prepared at the concentration of $5 \mathrm{mg} / \mathrm{mL}$ by diluting the powder in a mixture of dimethyl sulfoxide (DMSO) and $\mathrm{MeOH}$ 50:50 (v:v), while $\mathrm{QX}$ was prepared at the final concentration of $1 \mathrm{mg} / \mathrm{mL}$ in a mixture of $\mathrm{MeOH}: \mathrm{H}_{2} \mathrm{O}$ 90:10 (v:v).

DBV and QX stock solutions were stored at $-80{ }^{\circ} \mathrm{C}$ and $+4{ }^{\circ} \mathrm{C}$, respectively, for a maximum of six months. Standard 9 (STD 9) and quality control (QC) aliquots were prepared by independently spiking blank plasma from healthy donors with DBV stock solution and were stored at $-80^{\circ} \mathrm{C}$ for a maximum of six months. Calibration ranges and QC concentrations, chosen considering literature-derived data [7], are reported in Table 1. IS working solution was prepared before each analytical session by diluting $5 \mu \mathrm{L}$ of QX stock solution (final concentration: $1250 \mathrm{ng} / \mathrm{mL}$ ) and $40 \mu \mathrm{L}$ of phosphoric acid (final concentration: $1 \%$ ) in $4 \mathrm{~mL}$ of $\mathrm{H}_{2} \mathrm{O}: \mathrm{MeOH} 50: 50$ (v:v).

Table 1. Drug concentration in standards (ULOQ and LOQ) and quality controls (QCs).

\begin{tabular}{cccccccc}
\hline $\begin{array}{c}\text { Conc. } \\
\text { (ng/mL) }\end{array}$ & $\begin{array}{c}\text { ULOQ } \\
\text { (STD 9) }\end{array}$ & QC H & QC M & QC L & $\begin{array}{c}\text { LOQ } \\
\text { (STD 1) }\end{array}$ & LLOQ & LOD \\
\hline DBV & 250,000 & 200,000 & 30,000 & 3000 & 976.6 & 488.2 & 122.1 \\
\hline
\end{tabular}

\subsection{Sample Preparation}

The extraction protocol consisted of protein precipitation followed by dilution. In detail, $50 \mu \mathrm{L}$ of IS was added to $50 \mu \mathrm{L}$ of plasma sample and $300 \mu \mathrm{L}$ of ACN:MeOH 50:50 (v:v). All the samples were then vortex mixed and centrifuged: $200 \mu \mathrm{L}$ of the supernatant was then diluted with $800 \mu \mathrm{L}$ of pure water. Finally, $5 \mu \mathrm{L}$ of L-arginine stock solution $(50 \mathrm{mg} / \mathrm{mL}$ in $\mathrm{H}_{2} \mathrm{O}$ ) was added to each sample before injection into the HPLC-MS system. The injection volume was $5 \mu \mathrm{L}$.

\subsection{HPLC-MS Instrument and Chromatographic Conditions}

The HPLC-MS instrument consisted of a Waters (Milan, Italy) 1525 binary pump, a degasser, a CTC PAL autosampler and an Altus ${ }^{\circledR}$ SQ detector (a single quadrupole mass spectrometer, Perkin Elmer, Milan, Italy). Empower 3 Pro ${ }^{\circledR}$ software (year 2007 version, Waters, Milan, Italy) was used for data processing. Chromatographic separation was performed on an Atlantis ${ }^{\circledR} \mathrm{T} 35 \mu \mathrm{m} 4.6 \times 150 \mathrm{~mm}$ column (Waters, Milan, Italy), protected by a SecurityGuard ${ }^{\circledR}$ with a C18 $(4.0 \mathrm{~mm} \times 3.0 \mathrm{~mm})$ pre-column (Phenomenex, Torrance, CA, USA) and maintained at $45^{\circ} \mathrm{C}$ using a column oven. Separation was obtained with a gradient (Table 2) of mobile phases A (MP-A, $0.05 \% v / v$ formic acid in HPLC-grade $\mathrm{H}_{2} \mathrm{O}$ ) and B (MP-B 0.05\% $v / v$ formic acid in HPLC-grade ACN). A volumetric "T-switch" was 
used to discard $70 \%$ of the flow from the analytical column, only allowing $300 \mu \mathrm{L} / \mathrm{min}$ to enter the MS source. The instrument was settled in positive electrospray ionization mode (ESI+) for DBV and IS (QX). The general mass settings are presented in Table 3.

Table 2. Chromatographic gradient of mobile phases A $\left(0.05 \% v / v\right.$ formic acid in HPLC-grade $\left.\mathrm{H}_{2} \mathrm{O}\right)$ and $\mathrm{B}(0.05 \% v / v$ formic acid in HPLC-grade acetonitrile).

\begin{tabular}{cccc}
\hline Time (min) & Flow (mL/min) & Mobile Phase A (\%) & Mobile Phase B (\%) \\
\hline 0.00 & 1.0 & 93 & 7 \\
1.80 & 1.0 & 93 & 7 \\
5.50 & 1.0 & 50 & 50 \\
6.50 & 1.0 & 5 & 95 \\
8.00 & 1.0 & 5 & 95 \\
8.10 & 1.0 & 93 & 7 \\
11.00 & 1.0 & 93 & 7 \\
\hline
\end{tabular}

Table 3. Summary of instrument settings and single-ion recording (SIR) conditions.

\begin{tabular}{ccc}
\hline & General Detector Settings \\
\hline Capillary Voltage & $3.00 \mathrm{kV}$ & \\
Source Temperature & $150{ }^{\circ} \mathrm{C}$ & \\
Desolvation Temperature & $400^{\circ} \mathrm{C}$ & \\
Nitrogen Desolvation Flow & $600 \mathrm{~L} / \mathrm{h}$ & \\
Nitrogen Cone Flow & $50 \mathrm{~L} / \mathrm{h}$ & \\
& & \\
Mass Parameters & Analyte-specific Settings & QX (IS) \\
\hline Quantification Trace $(m / z)$ & DBV & 513.1 \\
Cone Voltage $(\mathrm{V})$ & 909.3 & 50 \\
\hline
\end{tabular}

\subsection{Accuracy, Precision, Limits of Quantification, Detection and Dilution Integrity}

Accuracy and inter-day precision were evaluated, as requested by the FDA and EMA guidelines [24,25]. Six validation sessions were performed and three different QCs samples were quantified in duplicate during each analysis; intra-day precision was evaluated in five replicates during a single analytical session. Inter-day and intra-day imprecision were expressed as the relative standard deviation (RSD\%) from the nominal value at each QC concentration. Integration was performed considering peak areas. The Upper Limit of Quantification (ULOQ) corresponds to the highest point (STD 9) of the calibration curve; the Limit of Quantification (LOQ) is the lowest amount (STD 1) of each calibration curve; the Lower Limit of Quantification (LLOQ) and the Limit of Detection (LOD) were considered as the lowest dilution of STD 1, which yielded a signal-to-noise ratio higher than 10 and 3, respectively. Moreover, the LLOQ was tested in five intraday and interday replicates in order to test percentage bias and RSD\% lower than 20\%. Dilution integrity was evaluated by analyzing a QC sample prepared at a concentration of $500 \mathrm{mg} / \mathrm{mL}$ (twice higher than the STD 9) after a 3-fold dilution in 5 intra-day replicates.

\subsection{Recovery and Extraction Efficiency}

Recovery was estimated during six validation sessions at high, medium, and low concentrations by comparing peak areas obtained from the injection of samples spiked with analytes before the extraction (pre-spike) with those originated from the chemical mix spiked with analytes at the same concentration and not extracted. Extraction efficiency was measured through the comparison of pre-spike and other samples spiked at the same concentration after the extraction (post-spike). 


\subsection{Matrix Effect}

The matrix effect was evaluated by comparing the "post-spike" signal at high, medium, and low QC levels in 6 different blank plasma lots with those from the direct injection of the same concentration of analytes in a solution of water-ACN-MeOH (70:15:15 v:v:v), as described by Taylor [27]. Moreover, the IS-normalized matrix effect (IS-nME), defined as the difference in the "area of the analyte/area of IS" ratio between the same analyte concentrations in matrix extracts and in pure solvents, was evaluated as previously described [28,29].

\subsection{Carry Over}

Carry over was determined by injecting blank samples after the ULOQ calibration standard. Carry over signal in these samples had to be lower than 20\% of the LOQ for DBV and $5 \%$ for the IS.

\subsection{Stability}

Stock solutions' stability was tested after 3 months by comparing the peak areas of fresh stock solutions with old stock solution stored at $-80^{\circ} \mathrm{C}$, even considering the NSB phenomenon through the direct injection of diluted (1:1000 v:v) stock solutions in a UHPLC-PDA system (Acquity H-Class ${ }^{\circledR}$ system, Waters, Milan, Italy).

Conversely, the stability in human plasma was evaluated at three QC concentrations using the presented HPLC-MS method. Multiple aliquots of QCs were prepared, analyzed once, and then stored at $-80{ }^{\circ} \mathrm{C}$. The effect of "freeze-and-thaw" cycles on the stability of plasma samples was determined by analyzing 2 aliquots at each QC concentration, previously frozen at $-80^{\circ} \mathrm{C}$, thawed up to three times and then compared with freshly prepared/extracted QC samples; post-extraction autosampler stability was evaluated through the comparison between QCs analyzed immediately after the extraction and their reanalysis after 7 days in the autosampler $\left(10^{\circ} \mathrm{C}\right)$. The long-term stability of DBV in human plasma was tested up to 2 months at $-80^{\circ} \mathrm{C}$.

\subsection{Selectivity}

The method was tested for its ability to differentiate DBV and IS signal from endogenous components in the matrix or other sample components. Moreover, 12 antiretrovirals (rilpivirine, dolutegravir, cobicistat, darunavir, elvitegravir, atazanavir, tenofovir alafenamide, tenofovir, abacavir, lamivudine, zidovudine, and emtricitabine), 13 antihypertensives (atenolol, doxazosin, ramipril, telmisartan, amlodipine, sacubitril, indapamide, hydrochlorothiazide, valsartan, nebivolol, chlorthalidone, olmesartan, and clonidine), 4 antifungal drugs (fluconazole, voriconazole, posaconazole, and itraconazole), and 3 glycopeptide antibiotics (vancomycin, teicoplanin, and daptomycin) were added to blank samples and to a QC H in order to investigate the presence of interfering peaks at DBV and QX retention times (RTs) or the evidence of significant changes in their signal, respectively.

\subsection{Clinical Application}

The validated method was applied for routine use in TDM in patients who underwent treatment with two high $1500 \mathrm{mg}$ weekly doses of DBV, in the context of the observational study "Appropriatezza terapeutica della terapia antinfettiva" (ethical approval n. 0040388 23 April 2020). Fifty-two samples were received at the Laboratory of Clinical Pharmacology and Pharmacogenetics of the University of Turin for TDM, from 4 male patients who gave informed consent for the use of TDM data. The mean patients characteristics were: age of 65 years old (range 40-74), weight of $73.5 \mathrm{~kg}$ (range 70-85), BMI of 23.2 (range 19.4-25.5); all patients received two high $1500 \mathrm{mg}$ DBV doses in two weeks. For 2 patients, the reason for TDM was altered renal function and renal replacement therapy (eGFR before dialysis 6.2 and $17.4 \mathrm{~mL} / \mathrm{min}$ ), with osteoarticular meticillin-resistant Staphilococcus aureus (MRSA) infection: one underwent intermittent hemodialysis (IHD, 4 h every $2-3$ days, 24 samples, 6 weeks of TDM follow-up) and the other one continuous veno-venous hemodi- 
afiltration (CVVHDF, 4 samples, 3 weeks of follow-up). The other two patients were in treatment for osteoarticular MRSA infection with normal renal function (eGFR 107.5 and $113.8 \mathrm{~mL} / \mathrm{min}$ ). They underwent TDM in order to evaluate the overall exposure over a period of 8 weeks, considering the already-known AUC/MIC-dependent PK/PD effect for DBV $[19,20]$. Blood sampling in lithium heparin tubes for TDM purposes was scheduled before and after DBV infusion and then, for IHD, it was repeated before and after each dialysis session; all other samples were taken at variable timings, based on clinical needs.

\subsection{Incurred Sample Reanalysis}

In order to assess method reproducibility in real-life specimens, 32 incurred samples were reanalyzed after a period of storage at $-80{ }^{\circ} \mathrm{C}$ of $2-4$ weeks. According to EMA guidelines [24], percent deviation between the first and second analysis, compared with their mean value, had to fall within at least $\pm 20 \%$ for at least $67 \%$ of reanalyzed samples.

\section{Results}

\subsection{Specificity and Selectivity}

The mean retention time for DBV was $6.58 \mathrm{~min}( \pm 0.05)$, while IS retention time was $8.36 \mathrm{~min}( \pm 0.05$; Figure 1$)$. As expected, blank plasma samples, as well as samples containing drugs used for selectivity testing, presented no interfering peaks at the corresponding analytes' retention times (Supplementary Figure S1). No significant ion suppression was observed in all the high QC samples spiked with the drugs used for selectivity testing, confirming the robustness of this method for potentially interfering concomitant drugs.

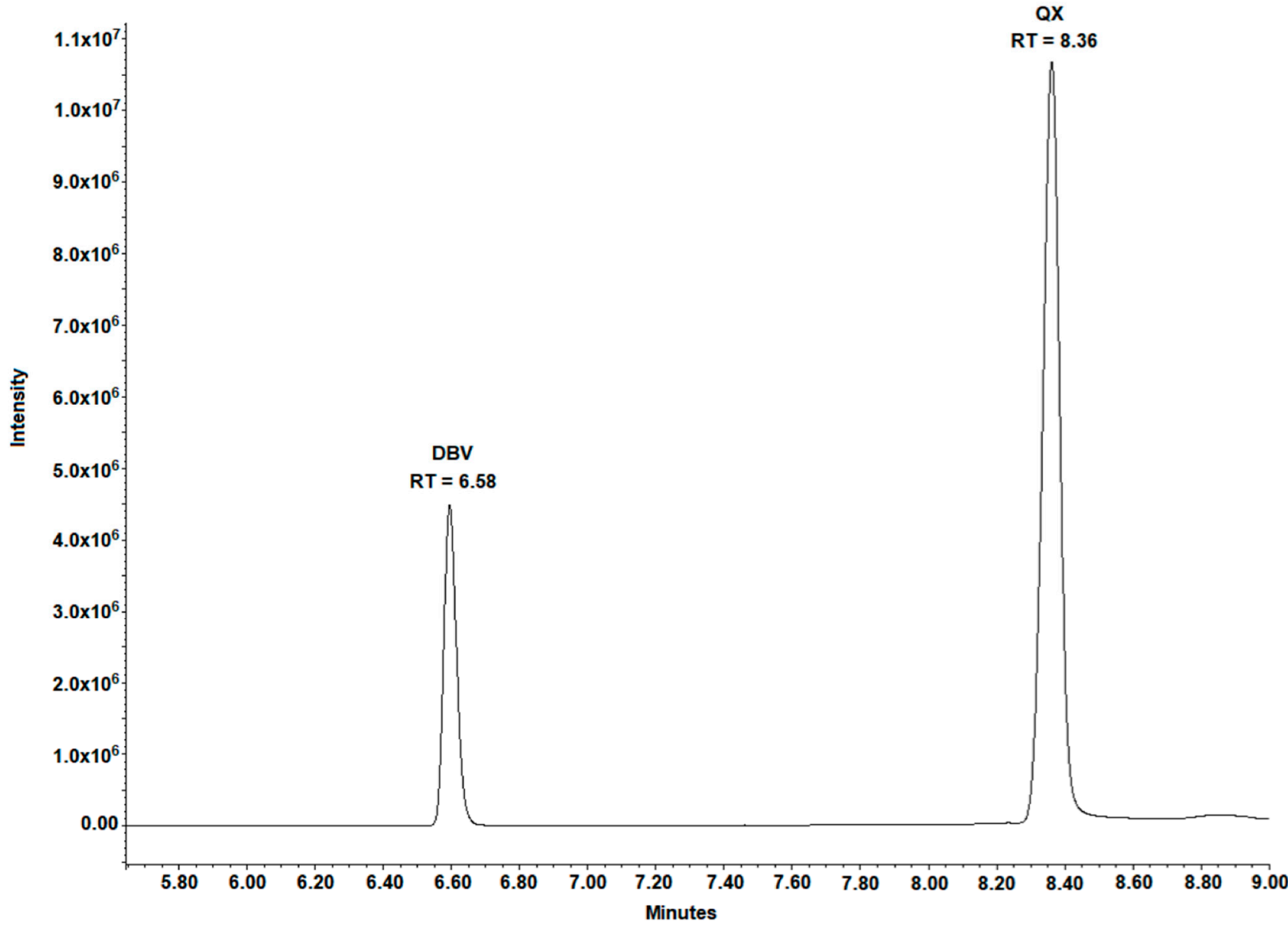

Figure 1. Dalbavancin (DBV) and internal standard (QX) chromatographic peaks and retention times (RT), resulting from the injection of the standard 9 (the highest point of the calibration curve), in presence of all the tested potential interfering drugs. 


\subsection{Accuracy, Imprecision, Limits of Quantification, Detection, Dilution Integrity}

Table 4 shows the accuracy and precision values for DBV at each different concentration. The mean obtained results for accuracy and imprecisions (intra- and inter-day) were as follows: $96.18 \%, 5.03 \%$ and $9.09 \%$, respectively. Concerning LLOQ and LOD, the values for DBV were, respectively, $488.3 \mathrm{ng} / \mathrm{mL}$ and $122.1 \mathrm{ng} / \mathrm{mL}$ (Table 1). Chromatograms were processed by peak area, and a quadratic force through the zero calibration model was used in order to balance a slight saturation phenomenon. A weighting factor of $1 / X$ was used to ensure high accuracy at low concentrations. The regression coefficients $\left(\mathrm{r}^{2}\right)$ of calibration curves were all above 0.998 . The analysis of a high concentration sample in five replicates after 3-fold dilution showed good accuracy and precision (mean $174.2 \mathrm{mg} / \mathrm{L} ; 4.5 \%$ bias, RSD 6.2\%), demonstrating acceptable dilution integrity.

Table 4. DBV validation parameters at 3 quality controls levels $(n=6)$.

\begin{tabular}{cccccccc}
\hline Level & Accuracy \% & \multicolumn{2}{c}{ Imprecision (RSD\%) } & \multirow{2}{*}{$\begin{array}{c}\text { Recovery } \\
\text { (RSD\%) }\end{array}$} & $\begin{array}{c}\text { Matrix Effect } \\
\text { (RSD\%) }\end{array}$ & $\begin{array}{c}\text { IS-Normalized } \\
\text { Matrix Effect } \\
\text { (RSD\%) }\end{array}$ & $\begin{array}{c}\text { Extraction } \\
\text { Efficiency } \\
\text { (RSD\%) }\end{array}$ \\
\cline { 3 - 4 } & & Intra-Day & Inter-Day & & & & \\
H & 99.8 & 2.0 & 8.4 & $62.5(9.5 \%)$ & $-16.8(7.7 \%)$ & $-18.6(6.50 \%)$ & $75.1(9.4 \%)$ \\
M & 100.5 & 6.0 & 7.1 & $59.3(9.8 \%)$ & $-17.5(10.8 \%)$ & $-19.3(9.3 \%)$ & $71.8(10.8 \%)$ \\
L & 88.2 & 7.1 & 11.8 & $56.2(10.0 \%)$ & $-18.9(10.1 \%)$ & $-20.0(9.6 \%)$ & $69.2(11.6 \%)$ \\
\hline
\end{tabular}

RSD $=$ relative standard deviation

\subsection{Recovery, Matrix Effect, IS-Normalized Matrix Effect and Extraction Efficiency}

A detailed summary of all the validation parameters at each QC level is presented in Table 4 . Briefly, the mean overall recovery was low (59.3\%) but highly reproducible (RSD $9.9 \%$ ); a negative matrix effect was detected but still reproducible (mean value $-17.7 \%$; RSD 9.6\%); the mean IS-nME (-19.3\%) was comparable to the matrix effect, but with a slightly lower (RSD $8.6 \%$ ). Finally, the mean extraction efficiency was $72.0 \%$, but stable at different concentrations and samples (RSD 10.3\%).

\subsection{Carry Over}

In accordance with the FDA and EMA guidelines [24,25], carry over in the blank samples analyzed after the injection of the highest concentration standard resulted in a signal $<20 \%$ of the LLOQ for DBV and $<5 \%$ for IS. These data suggested the absence of significant carry over.

\subsection{Stability}

DBV was found to be stable (percent degradation $1.3 \%$ ) in stock solutions at $-80{ }^{\circ} \mathrm{C}$ for 3 months upon the addition of $0.15 \mathrm{mg} / \mathrm{mL}$ of L-arginine in the stock solution. Without the addition of L-arginine, a percent reduction of $33.1 \%$ was registered, probably due to the NSB to glass containers on the long period. Concerning stability in plasma, DBV was found to be stable both after three "freeze-and-thaw" cycles, 7 days at $10{ }^{\circ} \mathrm{C}$ in the autosampler and up to 2 months at $-80^{\circ} \mathrm{C}$ (Table 5). These data were in accordance with previous works [21].

Table 5. Overview of dalbavancin (DBV) stability data.

\begin{tabular}{|c|c|c|c|c|c|c|c|c|}
\hline & & \multicolumn{3}{|c|}{ Freeze-and-Thaw Cycles } & \multirow{2}{*}{$\begin{array}{c}\text { Autosampler } \\
\left(10^{\circ} \mathrm{C}\right)\end{array}$} & \multicolumn{3}{|c|}{$\begin{array}{l}\text { Long-Term Storage } \\
\left(-80^{\circ} \mathrm{C}\right)\end{array}$} \\
\hline & & 2nd & $3 r d$ & 4th & & 2 Weeks & 4 Weeks & 8 Weeks \\
\hline \multirow{2}{*}{ QC H } & Bias $\%$ & 2.8 & -1.3 & -4.2 & -1.2 & $-2.4 \%$ & -3.2 & -4.5 \\
\hline & RSD\% & 1.9 & 0.9 & 3.0 & 0.9 & $5.8 \%$ & 4.7 & 6.5 \\
\hline \multirow{2}{*}{ QC M } & Bias $\%$ & 2.1 & 0.6 & 28.9 & -27.3 & -3.8 & -4.6 & -5.1 \\
\hline & RSD\% & 1.5 & 4.3 & 17.9 & 22.4 & 6.1 & 8.2 & 7.5 \\
\hline \multirow{2}{*}{ QC L } & Bias $\%$ & -6.7 & -4.7 & -5.8 & -9.6 & -4.2 & -5.0 & -4.1 \\
\hline & RSD\% & 4.9 & 3.4 & 3.7 & 7.1 & 8.5 & 7.6 & 9.3 \\
\hline
\end{tabular}




\subsection{Analysis of Clinical Samples}

The TDM results in relation with sampling time from the first $1500 \mathrm{mg}$ DBV infusion are depicted in Figure 2. The two patients with altered renal function started TDM followup before the second dose: pre- and post-infusion samples were also missing at the second infusion for the patient with intermittent dialysis.

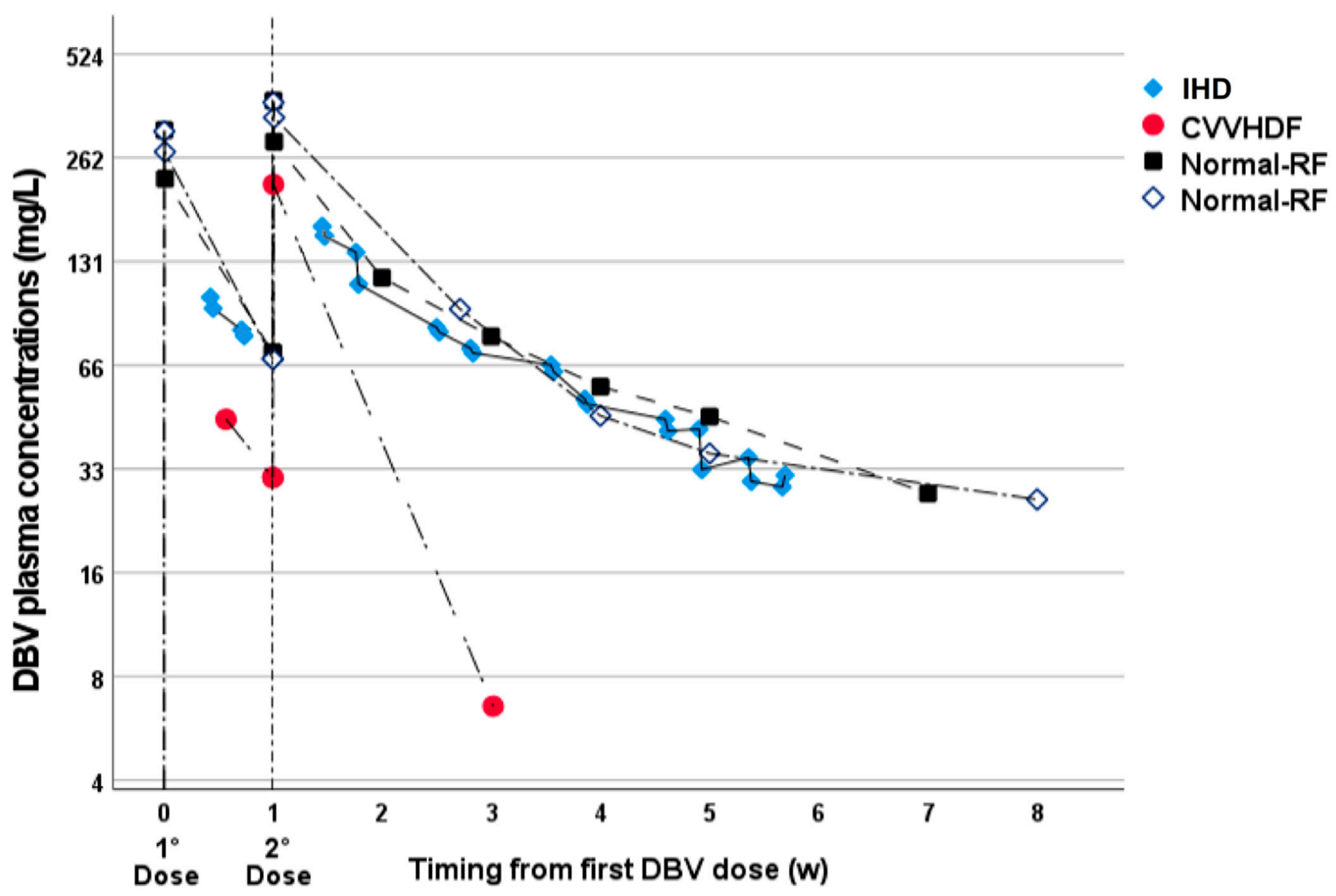

Figure 2. Dalbavancin (DBV) concentrations before and after the second $1500 \mathrm{mg}$ dose in four patients with normal or altered renal function and renal replacement therapy. TDM at the $\mathrm{C}_{\max }$ for the patient undergoing IHD was missed.

As is evident, the overall exposure appeared quite comparable between patients with normal renal function and the patient with intermittent dialysis. Conversely, CVVHDF was associated with a clinically relevant reduction in $\mathrm{DBV} \mathrm{C}_{\max }(219.6 \mathrm{mg} / \mathrm{L}$ vs. $381.8 \mathrm{mg} / \mathrm{L}$ mean concentration in patients with normal renal function) and half-life, leading to significantly lower overall exposure. In fact, the calculated mean DBV plasma concentrations based on the observed elimination curves after 2 weeks from the second infusion were, as follows: $70.3 \mathrm{mg} / \mathrm{L}$ for the patients with normal renal function, $68.4 \mathrm{mg} / \mathrm{L}$ for the patient with intermittent dialysis, and $6.7 \mathrm{mg} / \mathrm{L}$ for the one with CVVHDF.

This evidence was highly informative for a treatment switch in CVVHDF, still having a theoretical antibiotic coverage in this patient for the entire follow-up period: in fact, the theoretical breakpoint MIC in vivo, considering protein binding, can be approximated as $0.125 \mathrm{mg} / \mathrm{L}$ (EUCAST breakpoint MIC) $\times 0.07$ (literature-derived free-fraction in human plasma) $=1.786 \mathrm{mg} / \mathrm{L}$, in accordance with previous experimental data [20].

\subsection{Incurred Samples Reanalysis}

The results derived from the reanalysis of 32 samples all over the calibration range showed a mean overall deviation of $-7.3 \%$ (RSD 9.1\%). Only one sample (3.1\%) showed 
a percent deviation of $-23.2 \%$ after the reanalysis, falling out of the limits suggested by EMA guidelines.

\section{Discussion}

Nowadays, TDM has a crucial role in the management of those therapies characterized both by a narrow therapeutic index or by the need to constantly maintain adequate drug concentrations in blood, especially higher than the MIC, such as in the case of antibiotics.

Currently, very few data about DBV pharmacokinetics, and its off-label applications, have been reported in the literature. Furthermore, analytical procedures reported in the literature are sometimes discordant due to the chemical-physical properties of the molecule.

In this study, a new HPLC-MS method was validated in accordance with FDA and EMA guidelines, for DBV quantification in human plasma. This analytical protocol was designed in order to warrant the maximum adaptability to a clinical routine context, even in laboratories which lack expensive UHPLC-MS/MS instrumentation or cannot afford the use of isotope-labeled IS. In fact, to our knowledge, to date, only ${ }^{2} \mathrm{H}_{6}$-DBV is available on the market and it is extremely expensive (more than 1000 euros for $1 \mathrm{mg}$ ); moreover, for molecules characterized by a very high molecular weight, biprotonation with ESI+ and two chlorine atoms (polyalogens), the use of isotope-linked IS has to be managed with high caution due to the high risk of significant cross-talk when MS analysis with unit mass resolution is adopted (natural isotopes of the unlabeled analyte can partially overlap with the MS spectrum of the labeled IS). The choice of the concentration range used for the calibration curve and QCs was challenging; we focused on published data [7] reporting that $\mathrm{C}_{\max }$ of the two-dose regimen (1000 $\mathrm{mg}$ on day 1 followed by $500 \mathrm{mg}$ on day 8 ) can reach $278,000 \mathrm{ng} / \mathrm{mL}$, and chose $250,000 \mathrm{ng} / \mathrm{mL}$ for CAL 9 .

Since DBV has a strong affinity with plasma proteins, which could cause its coprecipitation during sample preparation, a previous work proposed the dilution of samples instead of protein precipitation [21]. Nevertheless, this approach could result in the suboptimal release of DBV from plasma proteins before LC-MS analysis which, other than drastically reducing analyte concentration in the final extract, could potentially increase the effect of NSB to the vials.

Our preliminary experiments evaluating the best precipitating solution indicated that $\mathrm{ACN}: \mathrm{MeOH} 50: 50$ (v:v) and $100 \% \mathrm{MeOH}$ yield superior performance in terms of analyte recovery compared to $\mathrm{ACN} \%$; in turn, $\mathrm{ACN}: \mathrm{MeOH}$ 50:50 (v:v) resulted in a clearer supernatant, with a reduced impact on analyte recovery and was therefore selected as the precipitating solution. The experiments regarding the effect of phosphoric acid revealed that the addition of $1 \%$ yielded a $20 \%$ increase in the analyte's recovery compared with samples without phosphoric acid, while higher percentages $(2 \%$ and $5 \%)$ did not result in a further increase in the chromatographic signal. Finally, comparative experiments to contrast DBV NSB showed that the addition of $1 \%$ Triton-X-100 had a similar effect to that of $5 \mu \mathrm{L}$ of L-arginine $(50 \mathrm{mg} / \mathrm{mL})$. Nevertheless, significantly higher background noise was observed in the chromatograms of samples treated with Triton-X-100, suggesting a possible increase in soluble contaminants within the extracts. For this reason, L-arginine was chosen as an additive to competitively decrease DBV NSB. Regarding the optimization of SIR traces, adducts with two and one proton were selected for DBV and IS, respectively. Concerning DBV, the effect of the presence of two chlorine atoms within its chemical structure caused the exact mass +2 daltons to be the most represented and stable ion to be recorded; therefore, the final selected SIR corresponded to the formula $(1814.6+2+2) / 2=909.3$.

All validation parameters were satisfactory according to the guidelines. Accuracy and precision evaluations totally fulfilled the validation purposes. The relatively low extraction efficiency could be partially explained by the extensive binding of DBV to plasma proteins. Other previous works which described DBV PK, using the LC-MS/MS technique for quantification, used acetonitrile for protein precipitation and did not report all the extraction efficiency and recovery data $[12,15,22]$, while another recent work evidenced poor recovery by using acetonitrile as a precipitating solution, in perfect accordance with 
our results [23]. In detail, only Zhu et al. [23] reported better recoveries (nearly 75\%) with $\mathrm{MeOH}$ as a precipitating solution, while Alebich-Kolbah et al. [21] chose to avoid protein precipitation, using an extreme dilution for sample preparation; nevertheless, this approach is viable only with instruments with a very high sensitivity and, moreover, could lead to problems in the long term due to the effect of remaining proteins in the final extract. Concerning the present study, the reproducibility of the extraction efficiency with ACN-: $\mathrm{MeOH}$ indicated that the impact on the analytical result remained acceptable, according to EMA and FDA guidelines, since the calibration curve and QC samples were prepared in the same matrix as the real-life specimens. Similarly, ion suppression was present but well reproducible among samples, even at different concentrations. The evaluation of IS-nME showed an even higher reproducibility, indicating that, despite the low chemical similarity between DBV and QX, this can be used as an acceptable IS, particularly according to EMA guidelines and previous works $[24,28]$. In any case, the described protocol can be easily modified in the future with the adoption of a stable isotope-linked IS or with a suitable analogue IS in order to further improve its analytical performance.

Interestingly, the quantification by single-MS spectrometry was possible even at high concentration ranges due to the high molecular mass of DBV (and therefore the lower molar concentration), the adoption of a strong dilution factor during sample preparation $(40 \times)$, and the use of a low injection volume with high mobile phases flow $(1 \mathrm{~mL} / \mathrm{min})$, providing further analyte dilution before the MS source. The use of a high flow was successfully adapted for use with ESI+ through the diversion of nearly $70 \%$ of the mobile phase flow, allowing a final flow $300 \mu \mathrm{L} / \mathrm{min}$ to enter the source, within the optimal range for ESI.

Unfortunately, to date, no acknowledged therapeutic ranges are known for DBV, since it shows AUC/MIC-dependent kinetics and because no concentration-dependent toxicity was described in the literature, although higher incidence of rash was described at very high doses. Currently, the protein-binding adjusted breakpoint MIC $(0.125 \mathrm{mg} / \mathrm{L}$ divided by $7 \%$ of free fraction $=1786 \mathrm{mg} / \mathrm{L}$ ) can serve as a reference value under which the growth of DBV-sensitive bacteria could be not successfully inhibited, as reported in some works and in accordance with experimental data by Leighton et al. [20,30]. Nevertheless, it is important to note that the vast majority of the staphylococci and streptococci isolates show MIC values abundantly lower than $0.125 \mathrm{ng} / \mathrm{mL}$ (most often 0.060 and sometimes $0.030 \mathrm{mg} / \mathrm{L}$ ); therefore, total DBV higher than 450 or $900 \mathrm{ng} / \mathrm{mL}$ could still be active.

The observed LLOQ and LOD allow one to even measure concentrations considerably lower than DBV MIC adjusted by plasma protein binding for the vast majority of bacterial strains, confirming the suitability of this method for the long-term evaluation of DBV concentrations. Moreover, the absence of significant carry over allows one to analyze both peak and trough concentrations within the same analytical session; it is worth noting that, in the majority of cases, the $1500 \mathrm{mg}$ dose administration resulted in $\mathrm{DBV} \mathrm{C}_{\max }$ higher than the ULOQ $(250 \mathrm{mg} / \mathrm{L})$. In these cases, accurate DBV quantification was possible after 3 -fold dilution, as confirmed by the dilution integrity experiments. Concerning stability, the observed data were crucial to prove DBV robustness in several analytical settings.

Compared with previous methods [12,15,21-23], the one proposed in this work provides an extremely cheap and reliable alternative, being based on HPLC and single-MS detection, without the use of labeled IS or unavailable analogues, but also provides a very thorough description of the validation process, with particular attention to the determination of the variability in extraction efficiency and matrix effect. Obviously, the drawback of the use of HPLC is a generally longer runtime for each injection, but this drawback is well balanced with the ruggedness of the validation process in human plasma and the potential for wider use in laboratories which lack UHPLC-MS/MS instrumentation.

The application of this method on incurred clinical samples for use in TDM showed a clinically relevant impact of CVVH on DBV exposure. On the other hand, IHD of 4 h every 2-4 days resulted in an overall exposure comparable with the one observed in patients with normal renal function. Based on these results, the application of a dual high dose of DBV appeared to be suitable for use with IHD, while particular attention should be paid to 
CVVHDF. Deepening this issue, a recent study showed evidence that the clearance of DBV can vary significantly among patients who undergo continuous renal replacement therapy, particularly based on the albuminemia [16]. The observed data in CVVHDF in this work are in good accordance with the ones from Corona et al., but in this case, we highlighted that the increase in DBV clearance in CVVHDF are high enough to reduce the overall exposure in a clinically significant manner, even in the absence of hypoalbuminemia, compared with patients with normal renal function.

In this setting, the presented method was shown to be clinically useful for the rapid identification of the CVVHDF-related reduction in DBV exposure, promptly guiding a treatment switch before reaching a DBV concentration below the theoretical protein-binding adjusted breakpoint MIC.

\title{
5. Conclusions
}

The described method was shown to be useful for DBV TDM purposes. Further future application on larger cohorts and in the context of clinical studies is guaranteed. Particularly, a thorough evaluation of the inter-patient variability in terms of DBV exposure in the common clinical practice is deserving of interest, particularly in light of the important identification of optimal therapeutic ranges.

Supplementary Materials: The following are available online at https:/ /www.mdpi.com/article/10 .3390 / separations8100189/s1, Figure S1, overlaid chromatogram of DBV at the LLOQ and in a blank sample spiked with the drugs tested for interference.

Author Contributions: Conceptualization, V.A. and A.D.N.; data curation, J.C., A.M. and A.P.; formal analysis, V.A., M.A. and E.D.D.V.; investigation, A.B., F.B., C.V., F.V. and F.G.D.R.; methodology, V.A., M.A., E.D.D.V. and A.D.N.; project administration, G.D.P., A.D.N. and A.D.; resources, G.D.P. and A.D.; supervision, A.D.N. and A.D.; writing-original draft, V.A., M.A. and E.D.D.V.; writing一review and editing, A.P. All authors have read and agreed to the published version of the manuscript.

Funding: This research received no external funding.

Institutional Review Board Statement: The study was conducted according to the guidelines of the Declaration of Helsinki, and approved by the Ethics Committee of the "A.O.U. Città della Salute e della Scienza" (protocol code 0040388 of the 23/04/2020).

Informed Consent Statement: Patients gave written informed consent to the publication of their TDM data.

Data Availability Statement: The data will be provided on request.

Acknowledgments: We thank CoQuaLab srl (Marco Simiele) for its kind technical counseling in method development.

Conflicts of Interest: The authors declare no conflict of interest.

Transparency Declarations: A.D. and G.D.P. are Shareholders of “CoQua Lab srl”.

\begin{abstract}
Abbreviations
DBV: dalbavancin; ABSSSI: skin and skin structure infections; MRSA: methicillin-resistant S. aureus; MIC: Minimum Inhibitory Concentration; AUC: Area Under the Curve; CL: clearance; $\mathrm{AUC}_{0-\infty}$ : AUC from time 0 to infinity; IHD: intermittent hemodialysis; CVVHDF: continuous veno-venous hemodiafiltration; NSB: non-specific binding; HPLC-MS: High-Performance Liquid Chromatography coupled to mass spectrometry; TDM: Therapeutic Drug Monitoring; ACN: acetonitrile; $\mathrm{MeOH}$ : methanol; QX: 6,7-dimethyl-2,3-di(2-pyridyl)quinoxaline; IS: internal standard; DMSO: dimethyl sulfoxide; STD: standard; QC: quality control; ESI: electrospray ionization; RSD: relative standard deviation; ULOQ: Upper Limit of Quantification; LOQ: Limit of Quantification; LLOQ: Lower Limit of Quantification; LOD: Limit of Detection; IS-nME: IS-normalized matrix effect; RT: retention time; $r^{2}$ : regression coefficient; IQR: inter quartile range.
\end{abstract}




\section{References}

1. US Food and Drug Administration. FDA approves dalvance to treat skin infections. Clin. Infect. Dis. 2014, 59. Available online: https:/ / pubmed.ncbi.nlm.nih.gov/25105184/ (accessed on 20 April 2020).

2. Klinker, K.; Borgert, S.J. Beyond vancomycin: The Tail of the Lipoglycopeptides. Clin. Ther. 2015, 37, 2619-2636. [CrossRef] [PubMed]

3. Lin, G.; Credito, K.; Ednie, L.M.; Appelbaum, P.C. Antistaphylococcal activity of dalbavancin, an experimental glycopeptide Antimicrob. Agents Chemother. 2005, 49, 770-772. [CrossRef] [PubMed]

4. McCurdy, S.P.; Jones, R.N.; Mendes, R.E.; Puttagunta, S.; Dunne, M.W. In vitro activity of dalbavancin against drug-resistant staphylococcus aureus isolates from a global surveillance program. Antimicrob. Agents Chemother. 2015, 59, 5007-5009. [CrossRef] [PubMed]

5. Smith, J.R.; Roberts, K.D.; Rybak, M.J. Dalbavancin: A Novel Lipoglycopeptide Antibiotic with Extended Activity against Gram-Positive Infections. Infect. Dis. Ther. 2015, 4, 245-258. [CrossRef] [PubMed]

6. EUCAST (The European Committee on Antimicrobial Susceptibility Testing). Breakpoint Tables for Interpretation of MICs and Zone Diameters. Version 10.0. 2020. Available online: http:/ / www.eucast.org (accessed on 25 November 2020).

7. Europe Commission. Annex I: Summary of Product Characteristics (Xydalba). Available online: https:/ / ec.europa.eu/health/ documents / community-register/2015/20150219130765/anx_130765_en.pdf (accessed on 25 November 2020).

8. Almangour, T.A.; Fletcher, V.; Alessa, M.; Alhifany, A.; Tabb, D. Multiple weekly dalbavancin dosing for the treatment of native vertebral osteomyelitis caused by methicillin-resistant staphylococcus aureus: A case report. Am. J. Case Rep. 2017, 18, 1315-1319. [CrossRef] [PubMed]

9. Almangour, T.A.; Perry, G.K.; Terriff, C.M.; Alhifany, A.; Kaye, K.S. Dalbavancin for the management of gram-positive osteomyelitis: Effectiveness and potential utility. Diagn. Microbiol. Infect. Dis. 2019, 93, 213-218. [CrossRef]

10. Boucher, H.W.; Wilcox, M.; Talbot, G.H.; Puttagunta, S.; Das, A.F.; Dunne, M.W. Once-weekly dalbavancin versus daily conventional therapy for skin infection. N. Engl. J. Med. 2014, 370, 2169-2179. [CrossRef] [PubMed]

11. Dowell, J.A.; Goldstein, B.P.; Buckwalter, M.; Stogniew, M.; Damle, B. Pharmacokinetic-pharmacodynamic modeling of dalbavancin, a novel glycopeptide antibiotic. J. Clin. Pharmacol. 2008, 48, 1063-1068. [CrossRef] [PubMed]

12. Buckwalter, M.; Dowell, J.A. Population pharmacokinetic analysis of dalbavancin, a novel lipoglycopeptide. J. Clin. Pharmacol. 2005, 45, 1279-1287. [CrossRef] [PubMed]

13. Dorr, M.B.; Jabes, D.; Cavaleri, M.; Dowell, J.; Mosconi, G.; Malabarba, A.; White, R.J.; Henkel, T.J. Human pharmacokinetics and rationale for once-weekly dosing of dalbavancin, a semi-synthetic glycopeptide. J. Antimicrob. Chemother. 2005, 55, ii25-ii30. [CrossRef]

14. Marbury, T.; Dowell, J.A.; Seltzer, E.; Buckwalter, M. Pharmacokinetics of dalbavancin in patients with renal or hepatic impairment. J. Clin. Pharmacol. 2009, 49, 465-476. [CrossRef] [PubMed]

15. Nicolau, D.P.; Sun, H.K.; Seltzer, E.; Buckwalter, M.; Dowell, J.A. Pharmacokinetics of dalbavancin in plasma and skin blister fluid. J. Antimicrob. Chemother. 2007, 60, 681-684. [CrossRef] [PubMed]

16. Corona, A.; Agarossi, A.; Veronese, A.; Cattaneo, D.; D'Avolio, A. Therapeutic drug monitoring of dalbavancin treatment in severe necrotizing fasciitis in 3 critically Ill patients: A grand round. Ther. Drug Monit. 2020, 42, 165-168. [CrossRef] [PubMed]

17. Pistolesi, V.; Morabito, S.; Di Mario, F.; Regolisti, G.; Cantarelli, C.; Fiaccadori, E. A guide to understanding antimicrobial drug dosing in critically Ill patients on renal replacement therapy. Antimicrob. Agents Chemother. 2019, 63, e00583-19. [CrossRef] [PubMed]

18. Vilay, A.M.; Shah, K.H.; Churchwell, M.D.; Patel, J.H.; DePestel, D.D.; Mueller, B.A. Modeled dalbavancin transmembrane clearance during intermittent and continuous renal replacement therapies. Blood Purif. 2010, 30, 37-43. [CrossRef]

19. Andes, D.; Craig, W.A. In vivo pharmacodynamic activity of the glycopeptide dalbavancin. Antimicrob. Agents Chemother. 2007, 51, 1633-1642. [CrossRef] [PubMed]

20. Leighton, A.; Gottlieb, A.B.; Dorr, M.B.; Jabes, D.; Mosconi, G.; VanSaders, C.; Mroszczak, E.J.; Campbell, K.C.M.; Kelly, E. Tolerability, pharmacokinetics, and serum bactericidal activity of intravenous dalbavancin in healthy volunteers. Antimicrob. Agents Chemother. 2004, 48, 1043-1046. [CrossRef]

21. Alebic-Kolbah, T.; Demers, R.; Cojocaru, L. Dalbavancin: Quantification in human plasma and urine by a new improved high performance liquid chromatography-tandem mass spectrometry method. J. Chromatogr. B 2011, 879, 2632-2641. [CrossRef] [PubMed]

22. Cavaleri, M.; Riva, S.; Valagussa, A.; Guanci, M.; Colombo, L.; Dowell, J.; Stogniew, M. Pharmacokinetics and excretion of dalbavancin in the rat. J. Antimicrob. Chemother. 2005, 55 (Suppl. 2), ii31-ii35. [CrossRef] [PubMed]

23. Zhu, D.; Ping, L.; Hong, Y.; Shen, J.; Weng, Q.; He, Q. Simultaneous quantification and pharmacokinetic study of five homologs of dalbavancin in rat plasma using UHPLC-MS/MS. Molecules 2020, 25, 4100. [CrossRef] [PubMed]

24. EMA. Guideline on Bioanalytical Method Validation. 2011. Available online: http://www.ema.europa.eu/docs/en_GB/ document_library/Scientific_guideline/2011/08/WC500109686.pdf (accessed on 15 May 2021).

25. FDA. Bioanalytical Method Validation. Guidance for Industry. Available online: https://www.fda.gov/downloads/drugs/ guidances/ucm070107.Pdf (accessed on 3 August 2018).

26. Dunne, M.W.; Zhou, M.; Darpo, B. A thorough QT study with dalbavancin: A novel lipoglycopeptide antibiotic for the treatment of acute bacterial skin and skin-structure infections. Int. J. Antimicrob. Agents 2015, 45, 393-398. [CrossRef] [PubMed] 
27. Taylor, P.J. Matrix effects: The achilles heel of quantitative high-performance liquid chromatography-electrospray-tandem mass spectrometry. Clin. Biochem. 2005, 38, 328-334. [CrossRef] [PubMed]

28. De Nicolò, A.; Cantù, M.; D'Avolio, A. Matrix effect management in liquid chromatography mass spectrometry: The internal standard normalized matrix effect. Bioanalysis 2017, 9, 1093-1105. [CrossRef] [PubMed]

29. Pensi, D.; De Nicolò, A.; Pinon, M.; Pisciotta, C.; Calvo, P.L.; Nonnato, A.; Romagnoli, R.; Tandoi, F.; Di Perri, G.; D’Avolio, A. First UHPLC-MS/MS method coupled with automated online SPE for quantification both of tacrolimus and everolimus in peripheral blood mononuclear cells and its application on samples from co-treated pediatric patients. J. Mass Spectrom. 2017, 52, 187-195. [CrossRef] [PubMed]

30. De Nicolò, A.; Stroffolini, G.; Antonucci, M.; Mula, J.; De Vivo, E.D.; Cusato, J.; Palermiti, A.; Cariti, G.; Di Perri, G.; Corcione, S.; et al. Long-term pharmacokinetics of dalbavancin in ABSSSI and osteoarticular settings: A real-life outpatient context. Biomedicines 2021, 9, 1288. [CrossRef] 